\title{
Probing the Mechanical Properties of Metal Wires at Small Scale by Microscopy
}

\author{
T. Scherer, ${ }^{*}$ S. Zhong, $* * * *$ and D. Wang* \\ * Institute of Nanotechnology, Karlsruhe Institute of Technology, Campus-Nord, D-76021 \\ Karlsruhe, Germany \\ ** Institute of Applied Physics and Center for Functional Nanostructures (CFN), Karlsruhe Institute \\ of Technology, Campus-Süd, D-76128 Karlsruhe, Germany
}

With the pace of miniaturization, investigating the mechanical properties of small objects and understanding their mechanism have been an urging task. However, developing mechanical tensile testing techniques and methodologies at micro- and nanoscale is challenging. Micro- and nanoscale tensile testing differs in comparison with other methods in that the interpretation of the data is relatively straightforward but the technical hurdles are high.

Two main challenges have to be mastered during tensile tests at small dimensions. One is the handling of extremely small specimens. The other is the requirements on force/displacement measurements. Here we use a Focus Ion Beam Microscope (FIB) with a micromanipulator to handle small specimens such as copper and silver sub-micro-wires. Force/displacement measurements were performed using Atomic Force Microscope (AFM) cantilevers. They are sensitive load sensors and can provide high accuracy of force measurement. By changing the different AFM cantilevers, a large range of force measurement can be achieved. The experiment set-up is shown in Figure 1. In our experiments the loading was applied continuously and the Scanning Electron Microscope (SEM) images were recorded in real-time using the movie tool. By analyzing the sequential snapshot images from the movie, the stress and strain curves could be drawn (Fig. 2).

In order to understand the mechanism of deformation and the mechanical properties at small scale, the investigations of the microstructure in materials before, after and during tensile tests are essential. Transmission Electron Microscopy (TEM) was performed to explore the microstructures at atomic scale. Figure 3 shows the microstructure change of silver wires before and after the tensile test. The results clearly show the stacking faults and twin boundaries are introduced into original single crystal silver wires. The mechanical properties of the metal nanowires strongly depend on their original microstructure and changes after the mechanical deformation.

Here, we demonstrate that combining different microscopy techniques (AFM, SEM, FIB, and TEM) is an efficient way to analyze the mechanical properties of metal nanowires. A novel and simple technique to measure the mechanical material properties of small metal wires was developed. This method can be easily applied to vast ranges of materials from normal metals to bio-materials such as feathers and hairs etc. TEM was used to investigate the microstructure change of metal nanowires before, after, and during mechanical tests. The results suggest that their mechanical properties strongly depend on their microstructures and microstructural changes due to deformation. 


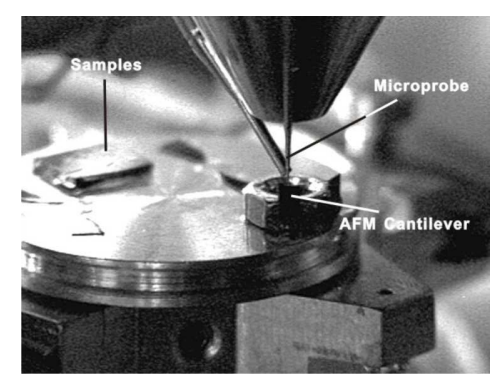

Fig 1. Experimental set-up: AFM cantilevers are attached to a metal block (here a simple screw nut) on a SEM stage by stable glue providing good electrical contact

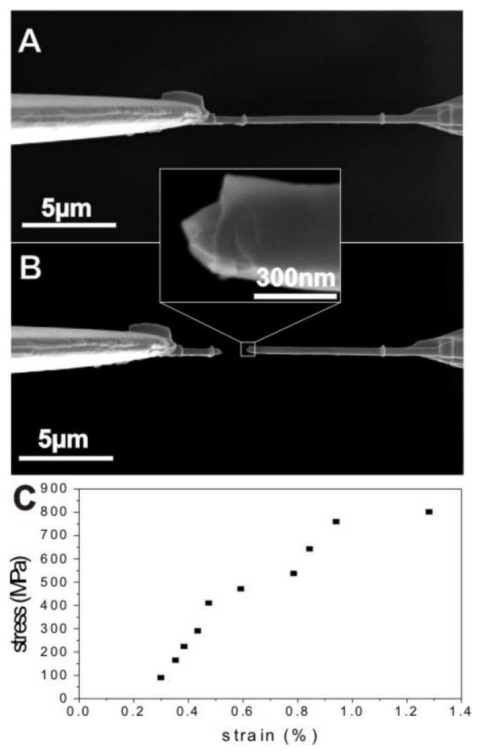

Fig 2. Top view of tensile test (silver wire). Before loading (A) and after failure (B), detail of broken wire (inset), stress and strain curve of silver wire (C).

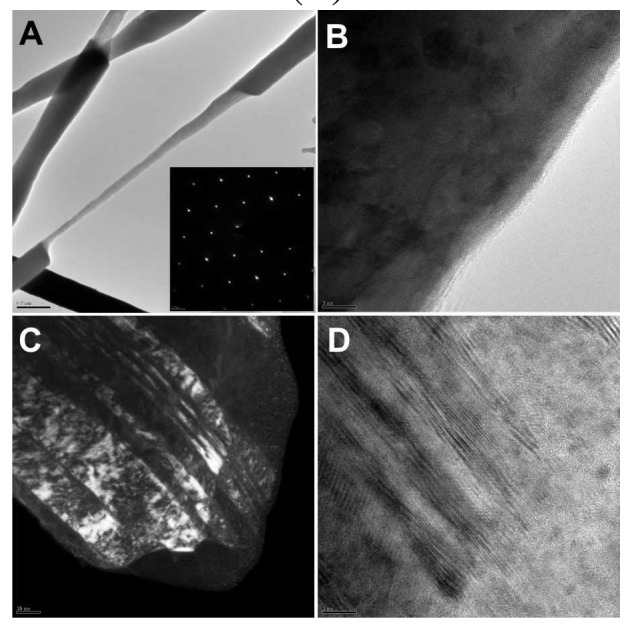

Fig 3. TEM analysis of silver wire microstructure before and after tensile test. (A-B) original silver wires. Overview of silver wire thinned by FIB and diffraction pattern (A). HRTEM image of silver wire, showing single crystalline microstructure without any defect (B).(C-D) Tested silver wire. Dark field of broken silver wire tip (C). HRTEM image of silver tip. Many stacking faults parallel to the axis of the silver wire can be found (D). 